\title{
INFORMATIVE AND FORMAL DESCRIPTION OF STRUCTURE DYNAMICS CONTROL TASK OF CYBER-PHYSICAL SYSTEMS
}

\author{
Sokolov Boris ${ }^{1}$, Potryasaev Semen ${ }^{1}$, Serova Elena ${ }^{2}$, Yury lpatov ${ }^{3 *}$, Yury Andrianov ${ }^{3}$ \\ ${ }^{1}$ St. Petersburg Institute for Informatics and Automation of the Russian Academy of Sciences (SPIIRAS), Russia \\ ${ }^{2}$ National Research University Higher School of Economics, Russia \\ ${ }^{3}$ Volga State University of Technology, Russia
}

The authors propose the informative and formal description of Structure Dynamics Control Task of Cyber-physical Systems (CPS). The main advantage of proposed poly-model multicriterion approach for the structure dynamics control tasks decision is that, in comparison with previously developed control models oriented on CPS simulation, proposed models allow to increase the efficiency, validity and, in general, the quality of the SD control CPS due to the original combination of their static and dynamic models.

Key words: Cyber-physical systems, Structure dynamics control, Integrated modelling and simulation.

\section{INTRODUCTION}

At present, large-scale production of microelectronic devices that can interact with physical world, perform calculations and exchange information on standard Internet protocols, rapidly penetrate into all spheres of human activity. The wide distribution of such devices is gradually change the habitual appearance of information systems due to the close integration of computing and physical processes, forming the information technology concept of cyber-physical systems (CPS) [1].

At the same time, CPS is generally considered as a new class of embedded systems, in which the concept of integration of computing, telecommunication, management resources with physical processes occurring in different subject areas, is realized at fundamentally new technological level. At the same time, the main elements of the CPS interact with each other using standard Internet protocols for forecasting, self-tuning and adaptation to changes. In general, CPSs are linked into spatially distributed structures, which form dynamic networks (DN).

One of realizations of CPS is Internet of Things. This is the concept of a computer network of physical objects equipped with computer and network modules for interaction with each other and with the external environment, excluding from the part of actions and operations necessity of human participation [2]. The Internet of Things is used in a wide range of applied areas: from organizing a person's daily life in a "smart home", to medical and transportation systems. One of the distinctive types of the Internet of Things is Industrial Internet of Things (or Industrial Internet), which is a multi-level system that includes sensors and controllers installed in the nodes and units of an industrial object, data transmission, remote control and control in automatic mode [3].Automated process control systems (APCS) can be considered as a precursor of Industrial Internet of Things. The main development directions are replacement of industry pro- tocols for devices interaction with Internet protocols and provision not only to the key nodes of the technological process by computers, but practically all the involved enterprise resources. In the industrial Internet of things, the orderliness of physical and computational processes is clearly pronounced; the purpose of the system's functioning has been formally defined. This purpose is production of high-quality competitive products. Spatial-temporal, technical, technological and financial constraints are identified. Further, in the article we will narrow the generalized class of CPS to its subclass - Industrial Internet, aimed at the release of physical products or information. Now, Cyber-physical systems, Industrial Internet of Things and number of related technologies are currently being considered in the framework of the Fourth Industrial Revolution, or "Industry 4.0".general concept. This terminology became widespread in 2011 as a result of German businessmen, politicians and scientists initiative as a tool of increasing the industry competitiveness through the enhanced integration of cyber-physical systems into production processes [4].

As a rule, the functioning of Dynamic Networks CPS depend on the influence of disturbing factors from external environment of targeted character (for example, violation of the information security regime) and / or non-targeted character (for example, errors, failures, extraordinary and emergency situations during the operation of these systems).

In other words, in practice, we are constantly faced with the structural dynamics of the CPS. In these cases, it is necessary to manage their structures (including management of the reconfiguration of the CPS structures) in order to increase (keep) the level of efficiency and capabilities of the CPS, or to provide the best conditions for degradation of these systems. 
At present, there are various options for managing of CPS structural dynamics. Among them, we can highlight, first of all: changing the methods, objectives of CPS operation, their content, the sequence of implementation under different conditions; moving in space of individual elements and subsystems of CPS; redistribution and decentralization of functions, tasks, control algorithms, information flows between CPS levels; using of flexible (abbreviated) CPS management technologies; reconfiguration of the structures of CPSs during their degradation.

The tasks of managing the structural dynamics of the CPS in its content belong to the class of tasks for structural and functional synthesis of the shape of the CPS and formation of appropriate programs for managing their development [4-6].

The main problem in solving tasks synthesis of technologies and programs for proactive management of CPS is as follows: determination of optimal control programs for basic elements and subsystems of CPS can be performed only after definition the list of functions and algorithms for information processing and control which should be realized in the specified elements and subsystems; in turn, distribution of functions and algorithms among elements and subsystems of CPS depends on the structure and parameters of control laws of these elements and subsystems. The difficulty of resolving this contradictory situation is aggravated by the fact that under the influence of various reasons structure of CPS changes in time.

To resolve this contradiction, it is proposed to use the main scientific results obtained in the currently developing theory of structural dynamics proactive control of complex objects including the class of CPS considered in this article [7-9].

\section{INTEGRATED MODELLING AND SIMULATION OF CONTROLLED STRUCTURAL DYNAMICS CPS}

Informative and mathematical formulation of the task of structure dynamics control of Cyber-physical Systems can be proposed as follows: let suppose that on a set of CPF nodes $A$, we can highlight on a set of information sources $A_{0}$, a set of transit nodes (repeaters/transponders) $A_{p}$ and a set of recipients (consumers) $A_{N}$. Bear in mind, that : $A_{0} \cup A_{\mathrm{p}} \cup A_{\mathrm{\Pi I}}=A ; A_{0} \cap A_{\mathrm{\Pi}}=\varnothing ; A_{0} \cap A_{\mathrm{p}} \neq \varnothing ; \quad A_{\mathrm{p}} \cap A_{\mathrm{II}} \neq \varnothing$. We will assume that the structure and parameters (characteristics) of the network change at discrete instants of time $\left(t_{0}, t_{1}, \ldots, t_{k}\right.$, which split the entire time interval $\left(t_{0}, t_{k}\right.$ ] (planning interval) into subintervals corresponding to the constancy of the structure. Assign number $\ell$ to an arbitrary interval $\left(t_{\ell-1}, t_{1}\right]$, where $\ell=1, \ldots k$. After that, we will assume that each element of the network (concrete CPS) is equipped with unified multifunctional hardware and software intended for storage, transmission and processing of data and information; the technical characteristics of the specified equipment are known: random-access memory (RAM) in each node $A_{j}$; computing power; capacity of communication channels between and nodes of the network $-\boldsymbol{\Psi}_{i j \ell}$, where $A_{i}, A_{j} \in\left\{A_{1}, A_{2}, \ldots, A_{n}\right\}$. At the next step for further formalization, we introduce the following variables: $y_{i \ell}, g_{j \ell}, z_{i \ell}$ - variables which characterize each respectively: volume of data and information that can be saved in $A$ node's memory in the $\ell$ interval, or processed at this node, or lost at this node (due to limited capacity of the CPS). In addition, let $\mathrm{q}_{\mathrm{ji}}{ }^{+}$- the set of network node numbers from which data and information are transferred to $A_{j}$ node on a time interval - $\ell$, and is the set of network node numbers into which data and information are transferred from the node $A_{j}$ on the time interval $-\ell$. Taking into account the foregoing, the static model of the controlled structural dynamics of the CPS, describing the processes of receiving, storing, transferring and processing information on the DN CPS will take the following form:

$$
\begin{gathered}
\alpha_{1} \sum_{j=1}^{m} \sum_{l=1}^{k} g_{i j}-\alpha_{2} \sum_{j=1}^{m} \sum_{l=1}^{k} z_{i j}=I_{1} \rightarrow \max , \\
x_{j l}=\sum_{i \in q_{j l}^{(-)}} x_{i j l}-\sum_{i \in q_{j l}^{(+)}} x_{i j l}+\left(y_{j l}-y_{j(l-1)}\right)+z_{j l}+g_{j l}, \\
0 \leq x_{i j l} \leq \psi_{i j l} ; 0 \leq y_{j l} \leq V_{j} ; 0 \leq g_{j l} \leq \varphi_{j l} ; z_{j l} \geq 0 .
\end{gathered}
$$

From the analysis of the constraint (2), it follows that the data and information $x_{j \ell}$, entered into $A_{j}$ node in $\ell$ interval of time can be transferred to other nodes, processed and stored in this one, or lost. When forming management options for CPS, search for the best alternative is carried out based on optimization of generalized indicator of CPS operation quality $I_{1}$. The first term in the relation (1)

$$
\left(I_{2}=\alpha_{1} \sum_{j=1}^{m} \sum_{l=1}^{k} g_{i j}\right)
$$

characterizes the total volume of data and information processed in the CPS, and the second term -

$$
\left(I_{2}=\alpha_{2} \sum_{j=1}^{m} \sum_{l=1}^{k} z_{i j}\right)
$$

the amount of data and information lost. From the analysis (1) - (3) it follows that, within the framework of the proposed formalization, task of planning functioning of DN CPS is reduced to multi-criteria task of linear programming (LP) large dimension. To overcome the problem of multi-criteria uncertainty in this task, we propose the variant of convolution for quality index vector of planning, based on the linear convolution of indicators. In this regard, in the expression (1) it is assumed: $\alpha_{1}+\alpha_{2}=1, \alpha_{1}, \alpha_{2} \geq 0$. Analysis proves that in spite of the large dimensionality of the planning task (1) - (3), the structure of constraint system defining in implicit form the set of feasible plans has a number of features that allow using special decomposition methods to build appropriate effective computational procedures based on methods of plan sequential improvement in LP task with bilateral constraints [5]. It is necessary to emphasize that in real functioning CPS consumers exchange among themselves heterogeneous information having a different degree of importance and 
urgency.

In this regard, it is proposed to further detail model (1) - (3) of the controlled structural dynamics of CPS already using the deterministic dynamic model(DM) of the following form:

$$
\begin{aligned}
& \dot{x}_{l}^{(\mathrm{o}, 1)}=u_{l}^{(\mathrm{o}, 1)} ; \dot{x}_{i j \rho l}^{(\Pi, 1)}=u_{i j \rho l}^{(\Pi, 1)} ; \dot{x}_{\tilde{j} \rho l}^{(\Pi, 2)}=u_{\tilde{j} \rho l}^{(\Pi, 2)} ; \dot{x}_{i j \rho l}^{(\Pi, 3)}=u_{i j \rho l}^{(\Pi, 1)} ; \\
& \dot{x}_{\tilde{j} \rho l}^{(\Pi, 4)}=u_{\tilde{j} \rho l}^{(\Pi, 4)} ; i, j, \widetilde{j} \in\{1, \ldots, m\},
\end{aligned}
$$

The constraints imposed on other control and phase variables are as follows:

$$
\begin{aligned}
& u_{l}^{(\mathrm{o}, 1)}\left[a_{(l-1)}^{(\mathrm{o}, 1)}-x_{(l-1)}^{(\mathrm{o}, 1)}\right]=0 ; \\
& 0 \leq u_{i j \rho l}^{(\pi, 1)} \leq c_{i j \rho}^{(\pi, 1)} u_{i j \rho l}^{(\pi, 3)} ; 0 \leq u_{\widetilde{j} \rho l}^{(\pi, 2)} \leq c_{\tilde{j} \rho l}^{(\pi, 2)} u_{\widetilde{j} \rho l}^{(\Pi, 4)} ; \\
& 0 \leq u_{i j \rho l}^{(\mathrm{n}, 3)} \leq u_{l}^{(\mathrm{o}, 1)} ; 0 \leq u_{\tilde{j} \rho l}^{(\mathrm{n}, 4)} \leq u_{l}^{(0,1)} ; \\
& \sum_{i \in q_{j l}^{(+)}} u_{i j \rho l}^{(\Pi, 3)}+u_{j \rho l}^{(\Pi, 4)} \geq 1 ; \quad \sum_{i \in q_{j l}^{(-)}} \sum_{\rho=1}^{P} x_{i j \rho l}^{(\Pi, 1)} x_{j \rho l}^{(\Pi, 4)} \leq \sum_{l=1}^{k} y_{j l} u_{l}^{(\mathrm{o}, 1)} \\
& \sum_{\rho=1}^{P} u_{i j \rho l}^{(\mathrm{n}, 1)}(t) \leq d_{i j l}^{(1)}(t) ; \sum_{\rho=1}^{P} u_{\widetilde{j} \rho l}^{(\mathrm{\Pi}, 2)}(t) \leq d_{\widetilde{j} l}^{(2)}(t) ; \\
& \psi_{i j l}=\int_{t_{l-1}}^{t_{l}} d_{i j l}^{(1)}(\tau) d \tau ; \varphi_{j l}=\int_{t_{l-1}}^{t_{l}} d_{\tilde{j} l}(\tau) d \tau ; \\
& x_{l}^{(\mathrm{o}, 1)}\left(t_{0}\right)=\dot{x}_{i j \rho l}^{(\mathrm{\Pi}, 1)}\left(t_{0}\right)=x_{\tilde{j} \rho l}^{(\Pi, 2)}\left(t_{0}\right)=x_{i j \rho l}^{(\Pi, 3)}\left(t_{0}\right)=x_{\tilde{j} \rho l}^{(\Pi, 4)}\left(t_{0}\right)=0 ; \\
& x_{l}^{(\mathrm{o}, 1)}\left(t_{f}\right)=a_{l}^{(\mathrm{o}, 1)}, \dot{x}_{i j \rho l}^{(\mathrm{\Pi}, 1)}\left(t_{f}\right), x_{\tilde{j} \rho l}^{(\Pi, 2)}\left(t_{f}\right), x_{i j \rho l}^{(\Pi, 3)}\left(t_{f}\right), x_{\tilde{j} \rho l}^{(\Pi, 4)}\left(t_{f}\right) \in \mathbf{R}^{1}
\end{aligned}
$$

where $x_{\ell}^{(0,1)}$ - is a variable, whose value is equal to the length of time interval for being the DN CPS in the $\ell$ - section of structures constancy; $x_{i j \rho \ell}^{(\pi, 1)}$ - amount of data and information of type $\rho$ transmitted from $A_{i}$ to $A_{j}$ in $\ell$ interval; $x_{\tilde{j} \rho l}^{(\pi, 2)}$ - the amount of data and information of type $\rho$ processed at $A_{\widetilde{j}}$ node on the $\ell$ interval; $u_{i j \rho l}^{(\mathrm{n}, 1)}(t), u_{\tilde{j} \rho l}^{(\pi, 2)}(t)$ - transfer rate of data and information type $\rho$ from $A_{i}$ to $A_{j}$ properly and the intensity of their processing in node $A_{\tilde{j}} ; x_{i j \rho l}^{(\pi, 3)}, x_{\tilde{j} \rho l}^{(\pi, 4)}$ auxiliary variables that are properly equal to the length of time interval from the moment of beginning of data and information of type $\rho$ transfer from $A_{i}$ to $A_{j}$ and time interval duration from the moment of beginning of data and information type $\rho$ processing (or storage) in the node

$$
A_{\tilde{j}} ; u_{l}^{(\mathrm{o}, 1)}(t), u_{i j \rho l}^{(\pi, 3)}(t), u_{\tilde{j} \rho l}^{(\pi, 4)}(t)
$$

- auxiliary control actions possessing the values from the set $\{0,1\}$. The indicators of work planning quality for $D N$ CPS are as follows:

$$
\begin{gathered}
I_{4}=\frac{1}{2} \sum_{l=1}^{k}\left[\left(a_{l}^{(\mathrm{n}, 1)}-\sum_{i, j, \rho} \gamma_{\rho}^{(1)} x_{i j \rho l}^{(\mathrm{n}, 1)}\left(t_{f}\right)\right)^{2}+\left(a_{l}^{(\mathrm{n}, 2)}-\sum_{j, \rho} \gamma_{\rho}^{(2)} x_{j \rho l}^{(\mathrm{n}, 2)}\left(t_{f}\right)\right)^{2}\right], \\
I_{5}=\sum_{l=1}^{k} \sum_{\rho=1}^{P} \sum_{j=1}^{m} \gamma_{\rho}^{(2)} x_{j \rho l}^{(\mathrm{n}, 4)}\left(t_{f}\right), \\
I_{6}=\frac{1}{2} \sum_{l=1}^{k} \sum_{j=1}^{m} \sum_{p=\tilde{j}+1}^{m-1}\left\{\int_{t_{l-1}}^{t_{l}}\left(x_{\tilde{j}}^{(\mathrm{n}, 4)}(\tau)-x_{p}^{(\mathrm{n}, 4)}(\tau)\right)^{2} d \tau+\left[x_{j \rho l}^{(\mathrm{n}, 4)}\left(t_{f}\right)-x_{\tilde{j} \rho l}^{(\mathrm{n}, 4)}\left(t_{f}\right)\right]\right\},
\end{gathered}
$$

where $\quad a_{l}^{(\mathrm{\Pi}, 1)}=\sum_{i=1}^{m} \sum_{j=1 j=1}^{m} x_{i j l} ; \quad a_{l}^{(\mathrm{\Pi}, 2)}=\sum_{j=1}^{m} g_{j l} \quad ; \quad$ indicator

allows quantifying the completeness of processing for received streams of heterogeneous data and information taking into account the given priorities; indicator (14) characterizes the total time delay of data and information in the DN because of their transfer, processing and storaqe at intermediate nodes. Known coefficients $\gamma_{\rho}^{(1)}$ and $\gamma_{\rho}^{(2)}$ specify the priority of data and information type $\rho$ when they are transmitted and processed. The indicator of the control quality (15) makes it possible to estimate the uniformity (non-uniformity) using of information-computational resources of CPS at each time [integral part of the functional (15)] and at the time $t=t_{f}$ [terminal part of the functional (15)]. Analysis of equations (5) - (12) shows that the coordination of the planning results obtained on the static and dynamic models, is carried out using the following variables $\mathrm{x}_{\mathrm{ijk}}, \mathrm{y}_{\mathrm{j \ell}}, g_{j \ell}$

\section{CONCLUSION}

The main advantage of proposed poly-model multi-criterion approach for the structure dynamics control tasks decision is that, in comparison with previously developed control models oriented on CPS simulation, proposed models allow to increase the efficiency, validity and, in general, the quality of the SD control CPS due to the original combination of their static and dynamic models.Preliminary analysis demonstrates that static models (1) - (3) have allowed to take into account those constraints which lead to phase constraints (data and information losses, bandwidth capability limitations) under dynamic simulation.Also, these models provide a narrowing of the initial data, under dynamic simulation, enumeration of which on the dynamic model would not be possible (in fact, an aggregated version of the technology for receiving and processing information was formed), and also allow, at first approximation, to take into account distribution and structural dynamics factors and estimate amount of data and information lost.

At the same time, a detailed description of distribution and processing processes of data and information with reference to specific time points in a static model is difficult. For this case, it is suggested to use a dynamic model. Dynamic interpretation of the CPS functioning allows forming and optimizing such parameters of the quality of controlled structural dynamics (CSD) of the CPS, which are difficult to describe with the help of static model.For example, indicators that assess the uniformity (non-uniformity) using of CPS resources throughout the control interval and at each current point of time. Dynamic description and interpretation of CSD processes makes it possible for them to involve extremely rich mathematical tools of optimal control theory.In general, the proposed multiple-model complex, which we are suggested in the paperillustrates the constructiveness of integrated modeling and simulation concept on the example of interaction between static and dynamic models of CPS. The site 
[9] presents the examples set of the developed models implementation in various subject areas (astronautics, robotics, shipbuilding).

\section{ACKNOWLEDGEMENT}

The results of the research were carried out with the financial support of the state-guaranteed order of the Ministry of Education and Science of the Russian Federation No. 2,3135.2017 / 4.6 (the 2 section of the paper), within the budget theme №№ 0073-2018-0003 (the 1 and 3 sections of the paper).

\section{REFERENCES}

1. TsvetkovV.Ya., (2017). Cyber-physical Systems. International Journal of Applied and Fundamental Research, 6-1, 64-65.

2. Chernyak L., (2012). Platform of Internet of things. Open systems. DBMS, 7,

3. Jeschke S. et al., (2017). Industrial Internet of Things: Cybermanufacturing Systems. Cham: Springer International Publishing. doi:10.1007/978-3-319-42559-7

4. https://www.din.de/blob/76902/e8cac883f42bf28536e7e8165993f1fd/recommendations-for-implementing-industry-4-0-data.pdf
5. Reznikov B.A. et al., (2013). Mathematical Support of Mobile Objects Management. Moscow: USSR Ministry of Education.

6. Moskvin B.V., (1987). Optimization of Data Transmission in a Packet-switched Computer Network. U: Trans. of All-Union conf. "Compac 87", Riga. 168171.

7. OkhtilevM.Yu., Sokolov B.V., \&Yusupov R.M., (2006). Intellectual technologies for monitoring and managing the structural dynamics of complex technical objects. Moscow: Nauka.

8. Potryasayev S.A., (2014). Synthesis of scenarios for modeling the structural dynamics of acs of active mobile objects. JournalofinstrumentEngineering, 57(11), 46-52.

9. http://litsam.ru 